\title{
Les Mutations de la représentation du divin au sein d'un groupe à vocation thérapeutique
}

Le cas de l'antoinisme

\section{Anne-Cécile Bégot}

\section{OpenEdition}

\section{Journals}

Édition électronique

URL : http://journals.openedition.org/assr/20222

DOI : $10.4000 /$ assr.20222

ISSN : 1777-5825

\section{Éditeur}

Éditions de l'EHESS

Édition imprimée

Date de publication : 1 septembre 2000

Pagination : 41-55

ISBN : 2-222-96695-7

ISSN : 0335-5985

\section{Référence électronique}

Anne-Cécile Bégot, « Les Mutations de la représentation du divin au sein d'un groupe à vocation

thérapeutique ", Archives de sciences sociales des religions [En ligne], 111 | juillet-septembre 2000, mis en ligne le 19 août 2009, consulté le 03 mai 2019. URL : http://journals.openedition.org/assr/20222 DOI : 10.4000/assr.20222

Ce document a été généré automatiquement le 3 mai 2019.

(c) Archives de sciences sociales des religions 


\section{Les Mutations de la représentation du divin au sein d'un groupe à vocation thérapeutique}

Le cas de l'antoinisme

Anne-Cécile Bégot

1 Cette étude se propose d'analyser, à travers le cas de l'antoinisme - un groupe religieux minoritaire centré sur des pratiques religiothérapeutiques - les évolutions de la représentation du divin qui ont accompagné les transformations de ce mouvement. L'antoinisme est passé d'une phase de domination charismatique avec à sa tête un prophète guérisseur, Louis Antoine, à une domination légale rationnelle où le successeur $\mathrm{du}$ prophète assure la routinisation du charisme, conformément au modèle de la succession du prophète chez Max Weber ${ }^{1}$. Au lendemain de cette succession, deux conceptions distinctes du divin apparaissent. Dès lors, pour la période contemporaine, on s'interrogera sur la gestion qui est faite de ce double héritage notamment en analysant les figures de Louis Antoine, l'expérience du sacré et les pratiques thérapeutiques.

\section{Origines et histoire de l'antoinisme}

Deux phases ponctuent l'histoire de l'antoinisme. La première voit l'avènement d'un homme, Louis Antoine, qui possède les différentes caractéristiques du prophèteguérisseur ${ }^{2}$. À cette première phase où prévaut le caractère extraordinaire de Louis Antoine, succède une phase postcharismatique, celle où Mère, la femme de L. Antoine, prend la succession du mouvement.

\section{Genèse d'un mouvement guérisseur}

3 Né au tout début du $\mathrm{XX}^{\mathrm{e}}$ siècle, en Belgique, l'antoinisme est impulsé par un homme: Louis Antoine (1846-1912). D'origine ouvrière, L. Antoine exerce diverses professions qui l'épuisent physiquement, notamment son travail aux mines de charbon. Vers l'âge de 
trente ans, il commence à ressentir différents symptômes dont il ne parviendra jamais à se défaire ${ }^{3}$. Ces précisions biographiques constituent un point fondamental pour comprendre l'histoire de l'antoinisme.

Préoccupé par sa santé et soucieux des "humbles », L. Antoine fonde avec ses amis un groupe spirite, Les Vignerons du Seigneur, où la guérison va progressivement occuper une place importante ${ }^{4}$. Des années 1890 à 1900, il recourt aux procédés habituels des magnétiseurs thérapeutes: sous l'influence des «esprits", les Docteurs Demeure et Carita, il a des « visions » des organes malades et prescrit des drogues telle que la liqueur Koene. Son attachement au spiritisme s'accentue, au début des années 1890, lorsque les funérailles de son fils, âgé de vingt ans, seront organisées par la Société Spirite de Liège.

Progressivement, la renommée de Louis Antoine s'élargit et les visites se multiplient. Les consultations thérapeutiques données dans le cadre des séances du groupe spirite vont peu à peu être dissociées de l'activité des Vignerons du Seigneur, pour être dévolues à Louis Antoine. À partir de 1900, ce dernier cesse toute activité professionnelle et agrandit le local où se tiennent les réunions du groupe ${ }^{5}$, localisé à Jemeppe-sur-Meuse (région de Liège). Ces nouvelles dispositions lui permettent de se consacrer entièrement à la guérison. Il reçoit les malades du lundi au vendredi, de sept heures à midi, et consacre ses dimanches aux séances du groupe spirite. D’après les sources de P. Debouxhtay, en 1900, Louis Antoine soigne entre cinquante et soixante personnes par jour, en 1901, une centaine ${ }^{6}$.

6 En 1901, se produit un événement décisif dans l'orientation des pratiques thérapeutiques de L. Antoine. Celui-ci doit comparaitre devant le tribunal correctionnel de Liège pour exercice illégal de la médecine. L'enquête engagée par des médecins aboutit à la conclusion suivante: L. Antoine est une menace pour la santé publique et le tribunal le condamne à une amende avec sursis. La clémence de ce jugement doit être envisagée au regard du contexte médical de l'époque. L. Antoine constitue une menace pour le corps médical, dont le discours est marqué par le thème de la pléthore et de l'encombrement de la profession ${ }^{7}$, mais il reste l'un de ces guérisseurs qui a contribué à la médicalisation des populations. Ses consultations sont gratuites, il oriente ses patients vers les médecins officiels et donne aux plus démunis l'argent nécessaire pour l'achat de médicaments ${ }^{8}$. Le jugement rendu par le tribunal correctionnel de Liège signe, de manière implicite, la reconnaissance du rôle joué par les guérisseurs et l'embarras du corps médical vis-à-vis de la concurrence faite par ces thérapeutes.

7 Suite à cette condamnation, L. Antoine tente de s'allier à la profession médicale. Il fait paraître une annonce, dans un journal local, pour proposer à des médecins de s'associer à lui. Ainsi, dans Le Messager, journal spirite, on pouvait lire l'annonce suivante: "Nous apprenons que M. Louis Antoine, de Jemeppe-sur-Meuse, se propose d'établir chez lui un dispensaire dirigé par des médecins diplômés. Lui écrire à ce sujet. Les praticiens belges désireux de s'initier à la pratique du magnétisme curatif peuvent également compter sur une clientèle assurée en dehors du dispensaire. La population de Jemeppe est de 10000 habitants et les communes voisines sont très populeuses ${ }^{9}$ ».

8 L'échec de cette tentative de collaboration et la condamnation du tribunal correctionnel de Liège conduisent $\mathrm{L}$. Antoine à abandonner le recours à des moyens médicamenteux. La lecture de l'ouvrage de Léon Denis, Dans l'invisible, l'amène à reconsidérer ses pratiques thérapeutiques. Désormais, écrit R. Vivier, l'un des biographes de L. Antoine, « la guérison magnétique n'exige ni passes, ni formules spéciales, mais seulement le désir ardent de 
soulager autrui, l'appel sincère et profond de l'âme à Dieu, principe et source de toutes les forces $^{10}$ ».

9 Ces événements marquent un tournant dans l'histoire de l'antoinisme. En 1906, la société des Vignerons du Seigneur disparaît. Elle signe la rupture de L. Antoine avec le mouvement spirite et sa volonté de privilégier la question éthique sur celle de l'expérimentation. Il abandonne l'évocation des esprits et se consacre à la morale. La foi du patient constitue alors le principal ressort de la guérison; elle se substitue à toute autre forme matérielle de guérison (cf. eau et papier magnétisés, médicaments,...). C'est à cette période que Louis Antoine apparaît à la tribune du Temple de Jemeppe-sur-Meuse, revêtu d'une robe noire. De sa chaire, il délivre ses "révélations " aux fidèles venus l'écouter. Il continue à recevoir individuellement les personnes venues le consulter et devient la figure centrale du culte. Le « Nouveau Spiritualisme » (1906-1910) voit alors le jour.

Éprouvé physiquement, Louis Antoine se retire de la vie publique en mai 1909. Durant cette retraite, il se soumet à une vie ascétique (jeûne et prière) et charge un adepte de faire la lecture de ses enseignements ${ }^{11}$. À son retour, le lundi de Pâques 1910, une foule importante l'attend. Là, il pratique l'Opération : du haut de la tribune, il impose ses mains sur l'assemblée de fidèles. Désormais, Louis Antoine ne reçoit plus individuellement ses patients; il considère que les "fluides" répandus sur l'assemblée, au moment de l'Opération, sont suffisants pour apporter la guérison.

11 Durant sa retraite, L. Antoine rédige le Couronnement de l'Æuvre révélée (1910). Ce texte marque la transition entre son précédent enseignement et le nouveau ${ }^{12}$, notamment en ce qui concerne la représentation du divin. Ainsi, dans l'un des textes sur l'évolution de l'homme, la représentation de Dieu est immanente. «Nous ne passons, écrit L. Antoine, d'une individualité à une autre plus élevée que par notre mérite car il n'y a pas d'inégalité possible dans l'amour (...) Cette évolution se continue jusqu'à la pureté absolue de l'être ; nous sommes arrivés là à la divinité, nous ne formons plus qu'une unité de l'ensemble, nous jouissons complètement de la toute puissance divine : restreints les uns dans les autres, nous sommes tous réunis dans un même amour, nous sommes Dieu ${ }^{13} ! »$. Dans d'autres textes, cette conception du divin est moins explicite et tend même à s'y opposer. Ainsi, les Dix Principes, l'un des textes antoinistes les plus connus, commence par « Dieu parle... ». Ou encore, à propos de ses révélations, Louis Antoine écrit, «je vous ai déjà enseigné que Dieu me disait, "Laissez-Moi pénétrer chez vous, ne Me repoussez pas ${ }^{14}$ " ".

On peut s'interroger sur cette double représentation du divin. En effet, de quelle manière la coexistence d'une figure transcendante et immanente du divin est-elle viable et surtout comment se manifeste-t-elle au sein du processus thérapeutique?

Pour Louis Antoine, toute "guérison" se mérite. La prière et le recueillement sont nécessaires mais insuffisants pour obtenir une guérison durable. L'élément essentiel de la cure thérapeutique consiste à envisager différemment la maladie. Ainsi, d'après L. Antoine, la souffrance « n'est pas le mal mais plutôt le remède... Plus nos imperfections sont nombreuses, plus imaginons-nous le mal. C'est l'importance que nous attachons à l'épreuve qui fait la souffrance et cette souffrance durera jusqu'à ce que nous ayons reconnu qu'elle est un bien. Arrivé là, l'homme s'écrie : 'sois béni, mon Dieu, de m'avoir donné la force de supporter mes épreuves'. Bien comprises, nos épreuves devraient nous charmer autant qu'elles nous martyrisent ${ }^{15} »$. Cette conception de la maladie permet l'élévation spirituelle et, à terme, la possibilité de ne faire qu'un avec le divin. La guérison antoiniste peut alors être comprise comme une nouvelle approche de la maladie assurant le salut de l'individu. 
La plausibilité de cette théodicée tient en partie à la possibilité de sublimer la souffrance. La croyance en une justice réparatrice, où la réincarnation permet de pallier les inégalités sociales ${ }^{16}$, en est l'un des ressorts. Prendre Louis Antoine comme modèle, en est un autre. La vie du prophète de Jemeppe-sur-Meuse, marquée par la souffrance physique (maladie stomacale lui provoquant des douleurs), l'abnégation (le décès de son fils est envisagé comme une réparation de la mort qu'il a donnée accidentellement à un soldat alors qu'il était réserviste), et l'ascétisme (L. Antoine se retirait de la vie publique pour se recueillir, jeûner et prier), incarne, aux yeux des antoinistes, la voie à suivre pour atteindre le divin. Elle est un modèle d'identification mais aussi ce qui fonde l'une des qualités charismatiques de Louis Antoine, celle de pouvoir entrer en contact avec le divin.

Ces deux versants de la figure de Louis Antoine permettent d'intégrer, en plus de la dimension éthique (acceptation de la souffrance comme un bienfait), la dimension collective à la cure antoiniste. Cette dimension collective se manifeste lors de l'Opération, lorsque Louis Antoine répand ses fluides sur l'assemblée de fidèles. Elle permet alors de transcender la souffrance, de rendre l'attente croyante plausible et supportable. En d'autres termes, le traitement socio-religieux de la maladie consiste avant tout à dépasser l'immédiateté de l'épreuve pour la transformer en un bienfait. Le cadre collectif de l'Opération en est l'un des ressorts mais il reste sous-tendu par cette dimension éthique où prévaut la réforme du comportement.

L'antoinisme a été mis en place en deux étapes. Au cours de la première, Louis Antoine est un médium-guérisseur qui discerne les symptômes et prescrit des remèdes avec l'aide des «Esprits ». La seconde étape commence aux alentours de 1906. Louis Antoine rompt avec le spiritisme et fait progressivement disparaitre le colloque singulier patient/thérapeute au profit d'une relation autrement singulière dans la mesure où la dimension thérapeutique s'exprime de manière collective, à travers les fluides répandus sur l'assemblée. À cette première dimension s'en ajoute une autre, une vision du cosmos et une éthique. L'individu est responsable de ses maux et il est le seul à pouvoir les dépasser. Ces deux orientations thérapeutiques sont coextensives à la double représentation du divin (transcendante et immanente). Dès lors, on peut s'interroger sur la gestion qui sera faite de l'héritage du Père Antoine.

\section{La succession de Louis Antoine}

17 «Désincarné », selon la terminologie consacrée dans le groupe, le 24 juin 1912, L. Antoine laisse derrière lui une œuvre naissante. À cette date, il existe deux Temples : celui de Jemeppe-sur-Meuse, consacré le 15 août 1910, et celui de Stembert (Belgique). Peu avant son décès, L. Antoine désigne sa femme, dite « la Mère », comme héritière de son œuvre.

18 Femme et analphabète, la Mère doit dépasser ces deux « handicaps " pour asseoir son autorité. Déjà, au lendemain du décès de son mari, elle disait : « quand Père est parti, Il ne m'avait rien dit, ne m'avait fait aucune recommandation, que celle de suivre Ses inspirations et j'ai eu beaucoup de tourments après son départ ${ }^{17}$ ».

19 Régulièrement, après la mort de Louis Antoine, paraissent dans L'Unitif, la revue antoiniste ${ }^{18}$, des textes visant à légitimer l'action de Mère. À titre d'exemple, on peut prendre cet article de décembre 1912 intitulé « Le premier interprète du Père ». Écrit par un fidèle, il justifie la présence de Mère à la tribune. « Mère, écrit ce fidèle, doit servir de transition ». Il poursuit son article en ces termes : «Lorsque le Père opérait visiblement, tous nous élevions notre pensée vers Lui directement, nous passions par son individualité 
terrestre pour L'atteindre en réalité, aujourd'hui qu'il est rentré dans le fluide pur, dégagé de la matière, comment Le toucher, nous qui sommes encore si matériels? Avonsnous bien compris les dernières paroles du Père lorsqu'il dit: "Je n'ai pas fait de testament, Mère est héritière de tout, c'est Mère qui me remplacera" ". Quelques lignes plus loin, l'auteur précise le sens de cet héritage : «Il (le Père) lui donne la mission divine de Le remplacer. Mère fait office de premier intermédiaire entre le Père et nous, Elle seule peut s'assimiler au fluide éthéré du Père, nous allons d'Elle à Lui, et Lui d'Elle à nous. C'est par Mère que le Père nous inspire. N'est-ce pas Elle que nous devons penser pour atteindre le Père ${ }^{19}$ ? ».

20 L'existence d'articles rédigés par des fidèles, au lendemain de la mort du Père, sur la place et le rôle tenus par Mère au sein du culte antoiniste, signe la volonté de contrôler toute crise de succession. Cette crise ne s'est pas déclarée parce que Mère n'a pas cherché à se substituer au Père : elle accepte d'en être l'intermédiaire voire l'intercesseur mais aussi parce qu'elle prend des mesures qui freinent toute forme de subversion du pouvoir au sein du mouvement. La principale d'entre elles est l'organisation d'un culte centralisé autour de la personne du Père ${ }^{20}$. Ainsi, au sein des Temples et dans les cabinets de guérisseurs, Mère fait apposer des tableaux comportant les inscriptions suivantes: « l'Enseignement du Père, c'est l'Enseignement du Christ, révélé à cette époque par la foi » et « Le Père Antoine, le Grand guérisseur de l'humanité pour celui qui a la foi ». Pour contrecarrer toute tentative d'appropriation du charisme du père fondateur, des articles paraissent dans L'Unitif pour rappeler le rôle des guérisseurs. Ainsi, dans le numéro de septembre 1913, il est indiqué que les "guérisseurs qui travaillent au nom du Père Antoine ne doivent pas perdre de vue qu'ils ne sont que des instruments; ils ne peuvent dire aux malades : 'pensez à moi et au Père', sous peine de falsifier leur travail, car c'est recourir à deux fluides... et en agissant ainsi, ils ne sont pas avec le Père Antoine ${ }^{21}$ ».

21 Au cours des années 1920-1930, Mère prend un certain nombre de mesures. Ainsi, entre 1925 et 1926, elle fait installer, devant la grande tribune, l'image du Père Antoine. " C'est à cette époque, note un fidèle, que l'on commence à se recueillir debout devant l'image du Père, en rentrant dans le Temple, alors qu'auparavant on s'asseyait dès qu'on entrait dans le Temple ${ }^{22}$ ». Au cours des années 1929-1930, elle autorise les desservants des Temples à faire l'Opération Générale à la grande tribune, mais elle fait lire l'annonce suivante, avant la cérémonie : « le Père fait l'Opération, suivie de la lecture des dix Principes. Celui qui a foi au Père trouvera satisfaction ", et sur la photo de Père installée à la tribune elle fait inscrire : «Le Père fait l'Opération ${ }^{23}$ ». De plus, elle exige, durant la lecture faite après l'Opération, que le desservant installé à la tribune s'assoie. Ainsi, il «s'efface derrière l'image du Père, ce qui est un symbole » car, selon elle, « le Père est tout ${ }^{24}$ ».

La volonté affichée par Mère de diviniser le Père conduit à un rapport plus immédiat au divin. Ainsi, l'introduction de la photo de Père, au sein des Temples, va servir à visualiser la figure divine qu'est censée incarner Louis Antoine. Ces changements n'ont pas toujours été bien compris par les fidèles car, comme le souligne B. Narinx, "bon nombre d'antoinistes ne savent pas très bien s'ils doivent prier Dieu, appelé le Père, dont Antoine est le prophète ou bien si Antoine est Dieu lui-même, comme le Christ était Dieu ${ }^{25}$ ». L'instauration de rituels tels que le baptême, la communion et le mariage ${ }^{26}$, rituels où le desservant transmet le «bon fluide du Père ", vise à inscrire le parcours des antoinistes dans un rapport au sacré. Quant à l'organisation de la fête du Père, le 25 juin, date de son décès, elle est l'occasion de rappeler sa présence au sein de chaque Temple, présence symbolisée par la manifestation de « fluides ». Par ailleurs, il faut indiquer que l'œuvre de 
Mère se caractérise par la multiplication de pratiques magiques (cf. déposer la robe antoiniste sur le lit d'un malade pour le couper de mauvais fluides) où la réforme du comportement importe moins que la recherche de bénéfices immédiats.

Les différentes modifications apportées par Mère avaient pour objectif de contrôler toute appropriation illégitime $\mathrm{du}$ charisme du Père Antoine par les desservants ou les guérisseurs en place. Elles ont ainsi permis d'éviter une crise de succession suite au décès de Louis Antoine sans toutefois être complètement légitimées de part et d'autre de la frontière franco-belge. Ainsi, au lendemain de la mort de Mère, en novembre 1940, la cultuelle antoiniste belge décide d'en revenir à un culte plus dépouillé, celui mis en place par Louis Antoine avant sa mort ${ }^{27}$. Les Temples belges vont être débarrassés des tableaux où figurent Père et Mère pour ne conserver que l'emblème antoiniste, «L'arbre de la Science de la vue du mal ${ }^{28}$ ». Quant à la principale cérémonie religieuse, l'Opération, elle n'est donnée que les quatre premiers jours de la semaine, et non du dimanche au vendredi comme cela se pratique en France.

La cultuelle antoiniste française s'inscrit dans la lignée de Mère. Il existe cependant une distinction majeure entre les écrits de L. Antoine et les « pensées » de Mère. Analphabète, Mère n'a pas pu rédiger les modifications qu'elle a apportées au culte antoiniste. Des fidèles s'en sont chargés. L'ensemble de ces modifications ainsi que diverses indications et prescriptions sont consignées dans les Tomes. Ces ouvrages sont présents dans chaque Temple mais inaccessibles aux fidèles ne portant pas le costume antoiniste. Circonscrite à un cercle d'initiés, l'œuvre de Mère ne peut prétendre à la même légitimité que celle de son mari (les livres du Père sont en vente dans le porche de chaque Temple). Alors que les écrits de Louis Antoine sont connus et reconnus par tous, ceux de Mère sont accessibles seulement à quelques-uns, les costumés. Dès lors, deux types de questions se posent. D'une part, quelle place occupe l'œuvre de Mère au sein de l'antoinisme français ? D'autre part, dans quelle mesure cette œuvre n'escamote-t-elle pas la dimension éthique préconisée par Louis Antoine?

\section{La gestion du rapport au divin au sein de l'antoinisme}

Le culte antoiniste français s'est constitué sur la base d'un double héritage, celui de Louis Antoine et celui de sa femme. À partir de cette situation, il est nécessaire d'envisager l'organisation des croyances et pratiques de guérison des adeptes. Les remarques et analyses qui suivent résultent d'une enquête de terrain réalisée à Paris, entre 1994 et 1997 29.

\section{Les figures du Père Antoine}

Le public antoiniste est constitué d'une population âgée, pour une grande part issue du catholicisme. Il regroupe des fidèles fréquentant régulièrement le culte, mais aussi une population fluctuante venant épisodiquement, lorsque le malheur se fait sentir. De manière générale, ces adeptes ont rencontré le culte antoiniste par l'intermédiaire d'une connaissance. Certains se contentent, en venant au Temple, de consulter un guérisseur et de repartir aussitôt après. D'autres s'investissent davantage, en assistant aux cérémonies, en consultant les livres du Père Antoine et en ayant un guérisseur attitré. Quant aux 
costumés ${ }^{30}$, ce sont des adeptes qui ont fait le choix de servir le Temple en effectuant un travail moral (accueil des adeptes dans le porche, lecture des ouvrages du Père Antoine, guérisseur,...).

La liberté laissée à chacun est, pour les adeptes, l'un des principaux attraits du groupe. Elle est aussi le principal obstacle pour socialiser les adeptes aux enseignements antoinistes. La diversité du public antoiniste, la difficulté de le fidéliser mais aussi les deux sources d'autorité que sont les écrits de Père et de Mère, dont l'une n'est accessible qu'aux costumés ${ }^{31}$, favorisent la présence de croyances multiples. Ainsi en est-il de la représentation de Louis Antoine qui, selon le discours des adeptes, prend trois figures différentes.

La première d'entre elles regroupe les fidèles pour qui L. Antoine est, au même titre que Jésus, une incarnation de Dieu. Les propos d'un desservant d'un Temple parisien rendent compte de cette représentation ${ }^{32}$. D'après lui, « Antoine et Jésus c'est la même face de Dieu. Il n'y avait pas de concurrence entre eux deux parce que le catholique que j'étais... J'avais évidemment des idées déjà un peu spéciales, en ce sens que je pensais que la réincarnation existait mais j'étais profondément attaché à la personne de Jésus et il y avait toujours une chose qui me hantait quand même. Je me disais, est-ce que je ne trahis pas la personne de Jésus? Est-ce que je ne trahis pas? Et donc je me suis posé pendant longtemps cette question jusqu'au jour où on m'a vraiment donné le... Je ne pourrais même pas vous expliquer comment, la certitude que non, que le Père Antoine n'était pas un faux prophète comme on dit, c'était bien la même face de Dieu. Ce jour-là j'ai plus eu aucun problème (...) J'avais non seulement la conviction mais je sentais, je palpais si je puis dire que la réincarnation ça existait et d'un autre côté j'étais en accord avec Jésus. Il $\mathrm{y}$ avait simplement deux mille ans de décalage entre les deux enseignements et ça fait que quand j'ai relu les Évangiles, je les ai relus avec un autre œil, je les comprenais autrement et j'ai compris pourquoi effectivement Jésus en tant que Jésus-homme c'était il y a deux mille ans et il avait donné un enseignement à l'époque et que le Père Antoine était là pour donner l'Enseignement d'aujourd'hui mais tout ça c'était la parole de Dieu ».

Le choix effectué par ce desservant n'a pas été immédiat mais progressif. De manière générale, les adeptes mettent plusieurs années avant de se détacher complètement de leur confession d'origine. Ici, la tension provoquée par des croyances « contradictoires » a trouvé une issue dans la création d'une filiation historique entre les personnages de Jésus et de Louis Antoine. Elle s'est aussi traduite par un choix, celui du caractère exclusif de l'antoinisme.

Dans le deuxième groupe d'adeptes, cette exclusivité n'existe pas et, ce, notamment parce que le choix entre l'antoinisme et le catholicisme n'a pas été effectué. La figure d'Antoine occupe la même place que Jésus, dans le panthéon des divinités; il est perçu comme un prophète, au même titre que Jésus, mais à la différence des fidèles du premier groupe, la frontière entre l'antoinisme et le catholicisme n'est pas établie ${ }^{33}$. Ces adeptes, pour la plupart costumés, continuent de fréquenter l'Église catholique tout en servant le culte antoiniste. Ces pratiques ne peuvent pas être considérées comme hétérodoxes dans la mesure où Louis Antoine cultivait une certaine ambiguïté à propos de l'exclusivisme religieux. En effet, même s'il considérait la doctrine catholique comme dépassée ${ }^{34}$, son attachement au principe du libre arbitre l'empêchait de préconiser l'allégeance à un dogme religieux. Le même constat peut être fait à propos du recours, par certains guérisseurs, à des prières catholiques. Ces emprunts ne sont pas préconisés par le groupe mais ils ne peuvent pas être considérés comme illégitimes puisque Louis Antoine écrivait 
« tout croyant doit réciter les prières que sa conscience lui dicte (...) C'est ainsi que nous devons comprendre la prière, qu'elle s'adresse à la Vierge ou à d'autres, si elle est sincère, c'est bien ; la conscience seule doit la sanctionner ${ }^{35}$... ».

31 Dans le troisième groupe d'adeptes, L. Antoine apparaît comme une figure spirituelle, une incarnation de l'essence divine. Parmi ces fidèles, pour la plupart non costumés, le recours à des croyances et pratiques issues de la tendance New Age, les amène à relativiser tant les formes d'organisations que les figures religieuses. Pour l'une de ces fidèles, qui fréquente le culte antoiniste depuis sept ans et pratique également le bouddhisme, L. Antoine est "quelqu'un de sage, quelqu'un qui a compris beaucoup de choses (...) Je ne pense pas que dieu c'est quelqu'un; dieu c'est une énergie. Je pense que dieu c'est quand on est arrivé à un certain état de perfection spirituelle. Y’a beaucoup de petits dieux qui forment un dieu mais je ne pense pas qu'il y a... Il doit y avoir une énergie qu'on appelle dieu mais dieu c'est pas une personne c'est pas quelqu'un avec une barbe c'est pas... On ne peut pas personnifier dieu de toute manière. Dieu c'est quelque chose qu'on ressent $»$.

Les différentes figures de Louis Antoine rendent compte, au sein de l'organisation religieuse, d'une certaine souplesse au niveau de la régulation du croire. Les antoinistes peuvent construire leur univers de sens en agençant des croyances et pratiques issues de traditions religieuses différentes - ce sont principalement le catholicisme et certaines croyances du New Age - sans pour autant faire l'objet de sanction. En ce sens, le principe du libre arbitre, principe fondamental dans les écrits antoinistes, est respecté. Ces croyances et pratiques n'en demeurent pas moins le signe d'une difficulté, celle de pouvoir actualiser les enseignements antoinistes aux temps présents. En effet, en dehors de la consultation d'un guérisseur, il n'existe pas de lieu permettant d'actualiser ou de commenter la doctrine du groupe.

Oscillant entre une conception immanente et transcendante du divin, les figures de L. Antoine traduisent la volonté, de la part de l'organisation religieuse, de ne pas chercher à délégitimer une part de son héritage, celle du Père et/ou celle de Mère. À partir de ce constat, il peut être intéressant d'envisager la manière dont est vécu le rapport au Père.

\section{Le ressenti : une expérience sacralisée}

Le Temple antoiniste est perçu par les adeptes comme un lieu sacré. Cette sacralisation tient avant tout au fait que l'Opération, met en scène, de manière symbolique, la présence du Père. Elle reproduit cette scène originelle où le lundi de Pâques de l'année 1910, Louis Antoine, installé à la tribune du Temple de Jemeppe-sur-Meuse, a répandu ses fluides sur l'assemblée.

Actuellement, le déroulement de cette cérémonie est le suivant. Après une brève annonce, le desservant du Temple et son acolyte sortent d'un cabinet de guérisseur pour prendre place, respectivement, à la grande et à la petite tribune. Parvenus à leur place et accompagnés des fidèles, ils se recueillent debout, en silence, dans la position de Forant (yeux clos et mains jointes à mi-hauteur du corps). Puis, après l'annonce de la lecture par le desservant, un adepte costumé prend place à la petite tribune pour lire des passages de l'enseignement de Louis Antoine. Cette lecture achevée, certains fidèles continueront à se recueillir, d'autres prendront place pour consulter un guérisseur tandis que d'autres partiront. La communion entre le desservant, son acolyte et le reste de l'assemblée est censée introduire la présence fluidique du Père Antoine. Cette présence n'est pas 
seulement de l'ordre de la représentation, elle est vécue et ressentie par les adeptes. Un sentiment de plénitude et de douce euphorie (cf. « habitus mystique ») prend place. Les manifestations fluidiques dépassent l'intériorité du sujet car certains disent les avoir vues (la plupart du temps c'est une tâche blanche qui est évoquée) tandis que d'autres les ont perçues (les bruits « étranges » entendus dans le Temple en sont un exemple).

L'expérience ressentie au cours de l'Opération, malgré son caractère individuel, reste inscrite dans un cadre collectif. La consultation du guérisseur permet de lui donner un cadre plus intime et davantage personnalisé. Cette consultation a lieu après l'Opération car pour une plus grande "efficacité », le consultant doit passer par cette phase de recueillement.

Les guérisseurs antoinistes donnent leur consultation au sein du Temple, dans des cabinets prévus à cet effet. Ce cabinet est une petite pièce d'environ quinze mètres carré. Aux murs sont accrochés cinq tableaux dont le plus imposant est une photo sur pied de Louis Antoine ${ }^{36}$. En accueillant le fidèle dans le cabinet, le guérisseur lui demande quelle grâce il souhaite obtenir. Cette invitation à la parole a une durée variable. Certains guérisseurs n'accordent pas plus de cinq minutes aux consultants tandis que d'autres leur consacrent entre vingt et trente minutes. La parole échangée n'est pas seulement l'occasion de mettre des mots sur les maux, elle participe à la construction d'un univers de sens. Indissociable des actes et pensées de l'être, la maladie s'inscrit dans une filiation à recomposer. Tout acte et toute pensée commis dans une vie antérieure peuvent être préjudiciables à la personne. L'unique porte de sortie à ce cycle est d'en accepter les conséquences. Tout l'art du guérisseur est d'apporter cette compréhension de l'épreuve sans heurter l'adepte. Plusieurs consultations sont parfois nécessaires. Certains adeptes ne l'accepteront jamais.

Au cours de leurs échanges au sein du cabinet, l'adepte et le guérisseur se trouvent debout, face au portrait du Père et tandis que le premier se raconte, le second prie. Avant de se quitter, le guérisseur propose d'adresser une prière au Père Antoine. Au cours de cette oraison, à peine audible pour le consultant, le guérisseur se fait intercesseur. Il peut ressentir les maux de l'autre : son corps devient un support d'identification ou le "passé sous silence ». D'autres sont pris d'un léger balancement d'avant en arrière et manifestent ainsi la présence du Père en leur sein. D'autres, enfin, ont un contact physique avec l'adepte comme si lui toucher la main ou l'épaule favorisait la transmission $\mathrm{du}$ « fluide ». De son côté, le consultant est également sensible à ce rapport qui s'instaure avec le guérisseur. Les fluides peuvent l'habiter, l'émotion le submerger, et ainsi lui procurer un sentiment de mieux-être.

39 L'échange symbolique opéré entre le guérisseur et le fidèle peut s'apparenter à une forme de "purification » dans la mesure où le premier est censé débarrasser le second de ses fluides négatifs. Cette expérience du sacré légitime toute autre forme de validation du croire. Ce n'est pas le contenu des croyances qui importe (le type de représentations du Père) mais cette expérience unique où l'adepte est amené à ressentir le Père en soi. L'importance accordée à cette expérience peut toutefois déboucher sur certaines dérives que l'organisation religieuse doit gérer.

Détenteurs d'un «charisme de fonction ${ }^{37}$ ", les guérisseurs agissent au nom de l'institution et sont reconnus par cette dernière. La difficulté à laquelle est confrontée l'organisation religieuse est de contenir le "charisme personnel privé » de certains guérisseurs. Entendue comme une qualité extraordinaire "avant toute intervention institutionnelle, hors d'elle ou au-delà d'elle ${ }^{38} »$, cette forme de charisme n'implique pas 
de novations importantes mais elle est constamment à la limite de l'illégitimité. La frontière entre le pouvoir reconnu aux guérisseurs, celui d'être les intercesseurs de forces supraempiriques, et celui dévolu à L. Antoine, le "grand guérisseur ", est ténue. Ainsi, des fidèles n'hésitent pas à envisager certains guérisseurs comme les acteurs de la guérison. La visibilité qu'ils acquièrent éclate aux yeux de tous. Par exemple, dans certains Temples, on repère très facilement que certains bancs, ceux attenant aux cabinets des guérisseurs, sont davantage remplis que d'autres. Cette situation peut conduire à modifier l'organisation du Temple. Ce fut le cas au Temple du Pré-Saint-Gervais (XIX ${ }^{\mathrm{e}}$ arrondissement de Paris). La renommée d'une guérisseuse n'était plus à faire. Chaque fois qu'elle donnait des consultations, trois bancs étaient assaillis par des adeptes. Le desservant du Temple a donc décidé de retirer le planning où étaient indiqués les noms des guérisseurs pour la semaine à venir. D'après lui, les adeptes doivent venir pour le Père et non pour un guérisseur particulier ${ }^{39}$. Cette mesure précise les frontières fixées par l'organisation religieuse : la figure du Père Antoine peut être investie différemment selon les fidèles mais le guérisseur, représentant légitime de l'organisation religieuse, doit rester un intercesseur des forces supraempiriques ; il ne peut s'approprier le charisme du père fondateur.

41 Le "charisme personnel privé " est difficile à gérer car il oscille entre une légitimité acquise et une légitimité "sauvage » mais aussi parce qu'il fonde la vitalité du groupe. Ainsi, dans l'exemple précité, une fois le planning retiré, les adeptes habitués à consulter cette guérisseuse sont venus de manière plus sporadique.

Les guérisseurs doivent constamment jouer sur deux tableaux : respecter les règles fixées par l'organisation religieuse (le rapport au divin s'inscrit dans un cadre précis) et contenir les attentes des fidèles, notamment en les amenant à les reformuler (cf. l'épreuve sert à l'amélioration de l'individu).

\section{La place de la guérison au sein de l'antoinisme}

La gestion socio-religieuse de la maladie a connu une certaine évolution dans l'histoire de l'antoinisme. Ainsi, alors que Louis Antoine considérait, à la fin de sa vie, que la prière était le meilleur remède pour une guérison durable, le groupe a ensuite connu certains changements: la guérison occupe une place périphérique et le recours aux moyens médicamenteux est banalisé. Deux raisons principales peuvent être évoquées : l'évolution de la médecine vers une plus grande efficacité thérapeutique et un incident survenu, dans les années 1950, dans un Temple du nord de la France ${ }^{40}$.

44 À la suite de cet incident, un feuillet apparaît dans les Temples antoinistes, sur lequel on peut lire les indications suivantes: «le Culte ne va pas sur le terrain de la science, notamment n'établit pas de diagnostic, ne prescrit ni ne déconseille aucun médicament, ni opération chirurgicale, ne fait ni passe, ni imposition des mains... Le culte, d'ordre spirituel, ne peut en aucun cas être mis en cause pour les soins du corps ».

Les conseils donnés aux guérisseurs vont dans le même sens. Ainsi, dans les Tomes, il est indiqué que «quand un malade est soulagé par le docteur (...) conseiller au malade de suivre les conseils du docteur. Ne pas conseiller au malade de quitter le docteur, ne pas prendre cette responsabilité, plutôt l'engager à y retourner. Seule la foi du malade peut l'engager à ne plus aller à la science ». Ou encore, «il ne faut pas que s'accrédite la légende selon laquelle le Culte interdirait à ses adeptes le recours au médecin ${ }^{41}$ ». 

les premiers étant dévolus aux médecins et les seconds aux guérisseurs antoinistes. Dans la pratique, cela se traduit par une banalisation du recours à la médecine allopathique ${ }^{42}$, et ce, même si certains antoinistes préfèrent les médecines douces (homéopathie, acupuncture, phytothérapie, ...) ou l'automédication. Le recours à la médecine allopathique ne s'effectue pas sans un regard critique sur l'institution médicale. Les rapports difficiles entre patient et personnel médical, la soumission à l'autorité médicale, la spécialisation du corps médical, le morcellement du corps humain, la logique économique qui prévaut au sein de la profession,... sont autant de motifs de récriminations qui conduisent les antoinistes à adopter une double démarche. Tout en ayant recours à la médecine allopathique, ils font appel au guérisseur antoiniste. Cette démarche est complémentaire; elle vise à pallier les "défaillances " d'une médecine considérée comme trop spécialisée, technicisée et/ou différenciée.

Dès lors, deux voies possibles sont offertes aux antoinistes. La première d'entre elles, qualifiée de voie "éthique », consiste à accepter l'épreuve car elle permet l'amélioration spirituelle de l'individu. Elle est généralement adoptée par les adeptes qui font de l'antoinisme leur religion d'appartenance. Dans la seconde voie, dite "magique », les fidèles s'inscrivent dans une logique où le respect d'un geste ou d'un rituel est censé provoquer la guérison ou la protection contre les mauvais « fluides ${ }^{43}$ ». Certains déposent la robe antoiniste sur le lit du malade. D'autres portent la photo de Père et de Mère dans leur portefeuille. D'autres encore inscrivent leur demande de guérison sur un morceau de papier qui, placé à la tribune, bénéficiera des fluides de l'opération. Quant à la consultation du guérisseur, elle consiste, selon les requêtes, à prier pour que le médecin soit « inspiré » au moment de faire son diagnostic ou de rédiger son ordonnance. Elle peut aussi viser à «stopper » les mauvais fluides. L'existence d'un public non costumé, venant épisodiquement au culte, notamment dans des moments de difficultés, peut exacerber ces pratiques de type magique qui donnent une certaine dynamique au groupe.

Les deux phases constitutives de l'antoinisme, celle du Père et de Mère, ont donné lieu à deux conceptions distinctes du divin et de l'efficacité thérapeutique. Alors que la première privilégie une conception immanente du divin et une éthique de la responsabilité, la seconde divinise la figure de Louis Antoine et favorise le recours à des pratiques magiques. La cultuelle antoiniste de France, reconnaissant ces deux héritages comme légitimes, gère les croyances et pratiques des adeptes de manière très souple, sans privilégier l'un plutôt que l'autre. L'absence de débat théologique autour de ces deux héritages facilite cette situation.

\section{NOTES}

1. MaX WEBER, Économie et société. L'organisation et les puissances de la société dans leur rapport avec l'économie, tome 2, Paris, Pion-Pocket, 1995, pp. 190-203.

2. Parmi les différentes caractéristiques du prophète dégagées par M. Weber, quatre peuvent être retenues :

Archives de sciences sociales des religions, 111 | juillet-septembre 2000 
1) le prophète est un personnage porteur de charisme, il est doué de qualités extraordinaires ;

2) le prophète développe une vision spécifique de la vie et du monde, une vision unitaire. Le salut des adeptes dépend de l'acceptation de cette vision du monde ;

3) les manifestations charismatiques du prophète, portées vers l'exceptionnel et l'extraordinaire, rejettent l'ordre du quotidien et en particulier la rentrée régulière des moyens de subsistance ;

4) enfin, le prophète usurpe son pouvoir. D'après M. Weber, « les prophètes n'assument pas leur mission parce que les hommes les en ont chargés. Cette mission, ils l'usurpent, c'est ce qui les caractérise ", in M. WEBER, Idem, p. 195.

On retrouve ces différentes caractéristiques, à des degrés divers, dans le personnage de Louis Antoine.

Ces quatre caractéristiques sont dégagées par Jean BAUBÉROT à partir d'une réflexion sur l'action menée par Nathan Söderblom, archevêque luthérien d'Uppsala, in " Nathan Söderblom : un réformateur religieux? ", Social Compass, vol. XXIX, n 1, 1982, p. 61.

3. D'après Pierre Debouxhtay, auteur de textes bien documentés sur l'antoinisme, L. Antoine était atteint d'une maladie d'estomac dont il ne parvint jamais à se guérir. Cette maladie, qui le faisait souffrir, «donnait à son visage un teint grisâtre et terne, décolorait le feu de son regard, entretenait en lui une nervosité toujours en éveil qui hachait sa parole et la faisait trembler ", in Pierre DEBOUXнтAY, Antoine le guérisseur et l'antoinisme. Les faits d'après des documents inédits, Liège, éditions F. Gothier, 1934, p. 53.

4. D'après les sources de Régis Dericquebourg, "les Vignerons du Seigneur guérissent les maladies, chassent les démons (mauvais esprits), ressuscitent les morts, s'entretiennent avec les disparus et donnent gratuitement ce qui leur a été donné gratuitement ", Souligné par nous, Régis DERICQUEBOURG, Les antoinistes, Tumhout (Belgique), éditions Brepols, 1993, p. 15.

5. L'inauguration de ce local se fera le 25 décembre 1900.

6. Pierre DEBOUXHTAY, op. cit., p. 160.

7. Carl Havelange, historien belge, a étudié le discours des médecins belges au XIX ${ }^{\mathrm{e}}$ siècle. Il souligne que la "pléthore", fut un thème récurrent dans le discours des médecins; il leur permettait notamment de construire leur identité professionnelle, in Carl HAVELANGE, « Histoire de la médecine. Pléthore et art de guérir. Le malaise des médecins liégeois au XIX ${ }^{\mathrm{e}}$ siècle ", Revue médicale de Liège, vol. XXXIX, n 19, oct. 1984, p. 677.

8. Au cours de son entretien avec le juge d'instruction, L. Antoine tient les propos suivants : «toutes les consultations que je donne sont gratuites; je n'exige aucune rétribution : seulement depuis six ans, à l'instigation de la société spirite "Les Vignerons du Seigneur" dont je fais partie, un tronc a été placé dans mon cabinet de consultation (...) j'ai la clef du tronc, je l'emploie pour assister les malheureux et notamment pour les aider à se payer les prescriptions que je leur fais ", cité par Pierre DEBOUXHTAY, op. cit., pp. 75-76.

9. Cité par Robert VIVIER, Délivrez-nous du mal, Éditions Grasset, Paris, 1936, p. 265.

10. Idem, p. 263.

11. Durant cette période l'antoinisme va connaître une dissidence. Le neveu de Louis Antoine, Pierre Dor, exerça ses dons de guérison, et devant le succès qu'il obtint, il fonda «L'école morale », dite « le Temple de la vertu. Culte de la miséricorde ». D’après Régis Dericquebourg, « il semble que les antoinistes n'aient pas manifesté d'animosité vis-à-vis de cette dissidence », in Religions de guérison. Antoinisme, Science Chrétienne, Scientologie, Paris, Cerf, 1988, p. 25.

12. D'après Louis Antoine, le Couronnement «nous instruit de tout ce qui nous est indispensable; il n'est pas seulement LE COURONNEMENT de mon enseignement, mais LE COURONNEMENT de l'œuvre accomplie, de tout ce qui a été révélé jusqu'à ce jour, par les prophètes qui sont venus rendre témoignage à la vérité ", Louis ANTOINE, Révélation par le Père, Poitiers-Ligugé, Éditions Aubin, 1979, p. 195. 
En s'inscrivant dans une lignée historique, L. Antoine souhaite apporter un nouvel éclairage aux précédents enseignements, un éclairage adapté à son époque.

13. Souligné par nous, Idem, p. XIII.

14. Ibid., p. 175.

15. Souligné par nous, Ibid., p. 11.

16. Issu du milieu ouvrier, Louis Antoine développa certains de ses enseignements autour de la justice sociale. Ainsi, à propos du bonheur, il tenait les propos suivants : «le richard... éprouve des souffrances morales d'autant plus terribles qu'il parait par sa richesse, être à l'abri d'autres, physiques ; mais elles (ces souffrances) ne se voient pas, elles gisent dans les replis de l'âme (...) Celui qui entrevoit la vie spirituelle. peut déjà comprendre que la mission du pauvre est cent fois, mille fois plus avantageuse que celle du riche (...) parce que sa fortune (au richard) le dirige vers les plaisirs qui ne font que cajoler toute faiblesse et nous amènent finalement des pensées bien noires qui alourdissent notre atmosphère. Comment, s'interrogeait L. Antoine, dans une telle situation, apprécier la richesse ?", Ajouté par nous, Ibid., pp. 40-41.

17. Tome 1. Les Tomes sont des ouvrages dans lesquels sont recueillies les «pensées » de Mère.

18. Revue qui est parue de septembre 1911 à août 1914.

19. J. F., (la signature ne comporte que les initiales) «Le premier interprète du Père ", L'Unitif, n -4, décembre 1912, pp. 2-3.

20. Il faut mentionner le fait qu'au lendemain de la mort du Père, le mouvement antoiniste est en pleine expansion. À la veille de la Première Guerre mondiale, le nombre de Temples belges passe à quatorze. Ce chiffre est doublé en 1940, l'année du décès de Mère. En France, durant la même période, seize Temples seront construits. De fait, Mère devait assurer la succession de son mari mais aussi gérer la construction de ces nouveaux Temples.

21. L'Unitif, septembre $1913, \mathrm{n}^{\circ} \mathrm{l}, \mathrm{p} .2$.

22. J. M. BOFFY, Louis Antoine et l'antoinisme. Données historiques rassemblées par le frère J. M. Boffy, document interne à l'antoinisme, non publié, p. 21.

23. On peut ajouter que Mère conseillait aux lecteurs, après leur lecture, de se dire intérieurement «Père, je vous remercie de m'avoir donné le bon fluide pour la lecture », Tome 2. A.

24. Tome 2. D.

25. Benoît NARINX, L'évolution du Culte antoiniste en Belgique, mémoire de licence de l'Université de Liège, 1986-1987, p. 69.

Dans des ouvrages écrits par des antoinistes, on retrouve cette confusion. Ainsi, pour GALAmus, Antoine est le "prophète de Jemeppe-sur-Meuse ", in Un message de liberté. L'Évangile de Jemeppesur-Meuse. Je suis dans le « Connais-toi », Toulouse, édition de L'Onde, 1949, p. 13.

Pour Jean-Marie DEFRANCE, Antoine est « l'apôtre de Jemeppe ", in Réveil. L'apôtre de Jemeppe et sa Révélation. "Tout est en Toi », Toulouse, éd. de l'Onde, 1932, p. 12.

Enfin, citons Léon MEUNIER, «Quand je salue Christ en Antoine, je ne fais pas un Dieu Antoine (...) Antoine est Christ comme, à un degré infiniment moindre, tout homme est "Christ",... », in... et la Lumière luit dans les ténèbres, Auxerre, Imprimerie du Bourguignon, 1923, p. 19.

26. Il faut indiquer que le Père Antoine considérait que ces rituels étaient dépassés. Ainsi, à propos du mariage, il disait, « aujourd'hui que nous avons acquis la lumière, il n'est plus question de pratiquer l'une ou l'autre loi pour nous unir. La Révélation les anéantit », Tomes 2.C.

27. D'après Benoît NARINX, les différences entre le public antoiniste français et celui de Belgique s'expliquent par un recrutement social différent. Les adeptes belges seraient issus de catégories sociales plus élevées que les adeptes français, in L'évolution du Culte antoiniste en Belgique, mémoire de licence de l'Université de Liège, 1986-1987, pp. 113-114.

28. D'après les antoinistes, cet arbre symbolise "l'incarnation». "Le tronc figure le "moi intelligent" c'est-à-dire le moi incarné. Les sept branches rappellent les sept péchés capitaux. Les deux yeux dans les branches sont la vue de ces péchés c'est-à-dire la "vue du mal". Les racines 
évoquent ce qui nous relie à la matière : l'intelligence", Régis DERICQUEBOURG, Les Antoinistes, Turnhout (Belgique), éd. Brepols, 1993, p. 87.

29. Sur Paris, il existe trois Temples antoinistes (Paris $\mathrm{XIII}^{\mathrm{e}}$, Paris $\mathrm{XVII}^{\mathrm{e}}$, Paris $\mathrm{XIX}$ ).

30. Tout fidèle peut, un jour, être amené à porter le costume antoiniste. Pour cela, il doit obtenir l'accord du desservant du Temple qu'il fréquente. Après cette entente préalable, il se fera faire un costume noir sur mesure (les femmes portent une robe noire qui descend à mi-mollet et un voile noir qui leur couvre les cheveux. Quant aux hommes, sur un pantalon noir, ils portent une sorte de redingote noire descendant un peu au-dessus de la cheville. Lors des enterrements ils portent un chapeau haut-de-forme noir), et sera reçu par le desservant dans son cabinet de consultation, suite à l'Opération. Cet entretien consiste à donner les conseils d'usage et à s'enquérir des disponibilités du costumé. Dans un premier temps, le costumé aura la charge de fonctions telles que la réception des fidèles dans le porche, l'annonce de l'Opération, la lecture, etc. Ensuite, le desservant pourra lui proposer de prendre un cabinet. Le desservant justifie cette nomination par le lien qui le rattache au Père Antoine. C'est au nom du Père, qu'il nomme les costumés guérisseurs. Ce recours au sacré, pour désigner les guérisseurs, justifie les nominations rapides mais aussi celles qui n'auront jamais lieu (un costumé peut ne jamais devenir guérisseur).

31. Il faut ajouter que les conseils et prescriptions donnés dans les Tomes ne sont pas faciles à mémoriser. En effet, l'adepte costumé ne peut en avoir une vue d'ensemble (quatorze Tomes), ni les consulter quand bon lui semble (la consultation se fait uniquement au Temple).

32. Ces propos ont été recueillis au cours d'un entretien réalisé lors de l'enquête de terrain.

33. À titre d'exemple, on peut citer cette fidèle costumée depuis sept ans. D'après elle, «l'enseignement catholique c'est la vérité et l'antoinisme c'est la vérité ; c'est Dieu, c'est Père donc tout. Christ, Seigneur Jésus ça a été un prophète de la parole de Père, la parole de Dieu qui n'a pas été comprise à ce moment-là et maintenant c'est Père qui est venu révéler en personne la parole de Dieu ".

34. Selon Louis Antoine, « on ne doit pas se rapporter à ce qui a été révélé il y a quelques mille ans pour justifier mon enseignement, une morale simple autrefois suffisait tandis qu'aujourd'hui, la science dont l'homme s'est pénétré ne lui permet plus de l'accepter (...) Ce qui était autrefois la lumière révélée par tous les prophètes, poursuit l'auteur, ne peut être pour celui qui se base sur mon enseignement que de l'obscurité ", Louis ANTOINE, Développement de l'Enseignement du Père, Poitiers-Ligugé, éditions d'Aubin, 1979, pp. 200-201.

35. Idem, pp. 61-63.

Signalons que les Tomes prêtent à la Mère les propos suivants : "notre pensée de demander une grâce au Christ après l'avoir demandée au Père est un doute », in Tomes 3. B. Il ne s'agit pas d'une injonction ou d'une prescription mais, comme bien souvent dans les Tomes, d'une recommandation.

36. Le deuxième tableau indique que «Tout fidèle qui fait payer sa prière n'est plus en accord avec la loi divine ", le troisième reprend «L'Auréole de la conscience », le quatrième comporte l'inscription suivante : «Le Père le grand guérisseur de l'humanité pour celui qui a la foi », et le dernier représente le symbole antoiniste, « L'arbre de la science de la vue du mal ».

37. Le charisme de fonction se traduit, selon Jean Séguy, par «une qualité exceptionnelle reconnue par une institution qui porte à plénitude par confirmation rituelle; celle-ci fonde la légitimité du pouvoir auquel elle prétend faire accéder ceux qui l'ont reçue; elle les oblige à reproduire les obligations articulées par l'institution, propriétaire du charisme de fonction; ce dernier tire sa légitimité en dernière analyse, de l'appropriation par une institution, d'un charisme personnel fondateur ", Jean SÉGUY, "Introduction. Le clergé dans une perspective sociologique ", VI Colloque du Centre de Sociologie du Protestantisme. Prêtres, pasteurs et rabbins dans la société contemporaine, Paris, Cerf, 1982, p. 40.

38. Jean SÉGUY, "Charisme, sacerdoce, fondation : autour de L. M. Grignion de Montfort », Social Compass, vol. XXIX, $n^{\circ} 1,1982$, p. 9. 
39. Ce problème était déjà présent dans la phase postcharismatique dans la mesure où Mère déplaçait certains desservants de leur Temple, et ce afin «qu'ils travaillent pour le Père et non pour les flatteries qu'on pouvait leur faire ", Tome 4. B.

40. Il s'agit du cas Phalempin. En avril 1955, une fillette est tombée dans de l'eau bouillante. Ses parents (Phalempin), antoinistes, l'ont conduite au Temple de Lille. Cette histoire va s'amplifier avec la rumeur publique. Après un examen médical, il est constaté que la fillette va bien physiquement. L'enquête judiciaire débouche sur un non-lieu.

41. Tome 2. D.

42. Comme le note R. Dericquebourg, à propos des "religions de guérison ", "l'incompatibilité fondamentale entre le traitement spirituel et le traitement biophysique a cédé la place à une coexistence de fait. Les fidèles et les praticiens consultent des médecins, se soumettent aux obligations légales dans le domaine de la santé (vaccinations, échographies de grossesse) », in Régis Dericquebourg, "La guérison par la religion. Perspective sociologique », Revue Française de Psychanalyse, $\mathrm{n}^{\circ} 3,1997$, p. 977.

43. Cette perspective est qualifiée de magique dans le sens où elle a peu d'incidence sur le comportement des adeptes. Elle peut être qualifiée de déterministe dans le sens où « si telles et telles conditions - de rites, de pureté régie par des règles de comportement - sont réalisées, l'effet attendu doit se produire ", in Françoise CHAMPION, "La nébuleuse mystique-ésotérique. Orientations psychoreligieuses des courants mystiques et ésotériques contemporains", in Françoise CHAMPION et Danièle HERVIEU-LÉGER, De l'émotion en religion. Renouveaux et traditions, Paris, Le Centurion, 1990, p. 44.

\section{RÉSUMÉS}

L'antoinisme est un groupe religieux minoritaire, d'origine belge, fondé sous l'impulsion de Louis Antoine. Apparu au tout début de ce siècle, ce groupe a pour particularité d'avoir centré ses pratiques autour de la guérison. La succession du "prophète» de Jemeppe-sur-Meuse a été assurée par sa femme dite Mère. Le culte antoiniste, toujours présent au sein du paysage religieux français, se distingue de son homologue belge en reconnaissant l'héritage légué par Mère. Il en résulte, au niveau de la représentation du divin, de l'expérience du sacré et des pratiques thérapeutiques, une certaine souplesse évitant de délégitimer l'un de ces héritages.

Antoinism is a minority religious group, of Belgian origin, based at instigation of Louis Antoine. Appeared at the very beginning of this century, this group has for peculiarity to have centred its practices around the cure. The succession of the "seer" of Jemeppe-sur-Meuse was insured by his wife called Mother. Cult antoiniste, always present within the French religious landscape, distinguishes itself from its Belgian homologue by recognizing inheritance bequeathed by Mother. It results from it, at the level of the representation of divine, of the experience of the sacred and the therapeutic practices, the certain flexibility avoiding of delegitime one of these inheritances.

El antoinismo es un grupo religioso minoritario de origen belga, fundado por Louis Antoine. Este grupo, aparecido a principios de este siglo del siglo XX, tiene como particularidad el centralisar sus practicas sobre la curacion. La sucesion del "profeta" de Jemeppe-sur-Meuse fué asegurada por su esposa conocida como la Madre. El culto antoiniste, aun presente en el «contexto » o 
paisaje religioso frances, se distingue de su homologo belga por el reconocimiento de la herencia legada por la Madre. Esto se traduce por una cierta flexibilidad en lo que concierne la representaclon de lo divino, de la experiencia de lo sagrado y de las practicas terapéuticas, evitando la perdida de la legitimidad de estas heréncias.

\section{AUTEUR}

\section{ANNE-CÉCILE BÉGOT}

Groupe de Sociologie des Religions et de la Laïcité - IRESCO 